\title{
Untapped Resources! Amazing Potential! TeNeT's Rural BPO Initiative
}

\author{
Saloni Malhotra ${ }^{[1]}$, P.Rathi ${ }^{[2]}$, Timothy A. Gonsalves ${ }^{[2]}$, Ashok Jhunjhunwala ${ }^{[2]}$, Thejus Giri $^{[3]}$ \\ 1 DesiCrew Solutions(P) Ltd. , \\ 2 TeNeT Group of IIT Madras, \\ 3 Nilgiri Networks(P) Ltd., Ooty
}

\begin{abstract}
DesiCrew is a unique Business Process Outsourcing (BPO) initiative that provides high volume data related services at an affordable cost from rural areas across India. The model provides cost cutting alternatives to urban clients and new sources of income and employment to the villagers, by leveraging Internet technology. The DesiCrew central team in Chennai manages training of rural workforce, distribution and monitoring of work, quality control and client interaction all remotely. In this paper, we describe the Rural BPO model and one specific application, data entry of forms. We describe the software, Pegasus, developed for this purpose and explain the management of the distributed workforce.
\end{abstract}

\section{Introduction}

In a village in South India, with a population of six thousand, where the primary occupation is weaving, Thenmozhi, a young graduate, runs an Internet kiosk business. With a single internet-connected computer, she provides a variety of services to the community that includes browsing, games, photography, education, and Desk Top Publishing among other BPO services. Over a period of a year, her income has grown and she has added two more computers to the kiosk. Today, with three PCs, she provides Business Process Outsourcing (BPO) services such as data entry and data conversion to clients located in distant urban centres.

In another village, Rosy, a young mother, runs a kiosk where she employs four people and provides BPO services such as Localization of content and Computer Aided Design (CAD). While these stories are undoubtedly incongruent with our

\footnotetext{
Ilease use the following format when citing this chapter:

Malhotra, S., Rathi, P., Gonsalves, T. A., Jhunjhunwala, A., Giri, T., 2007, in IFIP International Federation for Information Processing, Volume 24I, Home Informatics and Telematics: ICT for the Next Billion, eds. Venkatesh, A., Gonsalves, T., Monk, A., Buckner, K., (Boston: Springer), pp. $21-33$.
} 
predominant perceptions of rural India, indeed today BPO operations are making their way to the most remote and unlikely parts in the country.

As celebrated New York Times columnist, Thomas Friedman, observes in his latest book The World Is Flat [1], "all you need now is a global, web-enabled platform for multiple forms of sharing knowledge and work, irrespective of time, distance, geography and increasingly, language". As rural India increasingly becomes more connected, there is no reason that jobs cannot move - as they have from London to Bangalore - further to Vadalur, an unheard of village in the southern state of Tamil Nadu. Today there are approximately 15,000 Internet-enabled villages in India. With various efforts underway toward enabling rural connectivity, such as Mission 2007 and the Common Service Centre [2] scheme of the Government of India, this number is projected to reach 100,000 by 2008 . In this scenario, the potential for the rural BPO industry can only be expected to grow.

In this paper, this unique initiative of the TeNeT [3] of IIT Madras is described. First the business model is discussed in Section 2. This is followed by a description of the software used for one aspect of BPO in Section 4 . The paper is concluded with our future vision in Section 6.

\section{The Rural BPO Business Model [4]}

The TeNeT at IIT-Madras has worked over the past 26 months on a Rural BPO initiative that links urban clients with a rural workforce through the Internet kiosk network. This is now spun off as the company DesiCrew [5]. The team identifies and trains workers in rural areas in various skills relevant to the BPO industry. It liaises with urban clients and takes complete responsibility for the outsourcing and timely delivery of the projects undertaken, and ensures that quality standards are met. As a coordinating agency, the team protects the interests of both the clients and the kiosks. At the village-end it filters out unproductive kiosks from the system, and at the cityend it runs due diligence checks on the client to guard against fraudulent BPO activity.

This unique initiative has a portfolio of services that includes

1. Typing in English and regional languages,

2. Data entry operations, web and multimedia development and

3. Regional language translation.

More recently, engineering services such as $2 \mathrm{D}$ drafting and conversion of $2 \mathrm{D}$ to 3D for the manufacturing sector have also been introduced.

The model today runs on a smaller scale, with 3-4 seats in each center and an entrepreneur is responsible for hiring and managing the staff, ensuring that timelines and quality standards are adhered to, and managing daily operations. However a typical rural BPO center envisaged in the future would consist of 10 PCs running in two shifts. Each center would employ between 10-20 individuals. 


\subsection{Impacts and Benefits}

BPO activity in India is clustered around 5 main hubs today. These centers will continue to remain important in the future, but the industry is looking to expand to other locations for several reasons:

1. Newer locations would imply access to a larger workforce

2. Provide an opportunity to further reduce costs of operation

3. Acquire language-specific skills for the domestic industry

4. Mitigate overall business risk and ensure business continuity

5. Reduce the pressure on infrastructure being faced in the current locations

Rural areas can be attractive outsourcing destinations for the BPO industry due to the following reasons:

Access to available infrastructure: Currently 15,000 rural internet kiosks have been set up across the country. Even at a modest 10\%, 1,500 such centers could be utilized for the purpose of Rural BPOs.

Access to untapped and well trained work force: The model employs 10-20 individuals in each village. At 1500 villages with 20 employees, the rural $\mathrm{BPO}$ has the potential of bringing 30,000 youth into the workforce, considering the existing infrastructure.

Lower costs of operation: A reduction of 50-60\% in costs can be achieved using this model owing to the lower cost of living, real estate and overheads in a rural area. Bench strength at a lower cost: Leverage low cost resources to maintain mandatory bench size.

Improve skill levels at lower training costs: About $70 \%$ of Indian youth remain under/ unemployed in the first 3 years after graduation. Such resources can be trained at a lower cost in the rural BPO settings.

Scalability: Significant amount of time at DesiCrew is spent at how to leverage a workforce distributed across different villages. Tools, applications, processes and policy have been laid down to ensure rapid scalability.

Strong regional language skills can be leveraged for domestic outsourcing work including regional language call centers.

Presence of a entrepreneur: The existence of an entrepreneur running a rural BPO center ensures local knowledge of the location and culture. The entrepreneur is also a trusted entry point into the village.

DesiCrew's rural BPO model offers significant benefits to the rural population. With IT training, the youth in rural areas are exposed to skills that are highly valued in today's economy. As a result their productivity and incomes increase, and so also their personal confidence. The entire rural economy begins to thrive as more money flows into villages, allowing for more equitable economic growth at the national level. 
While the concept described above has been executed with encouraging results, the challenge is to scale it to thousands of villages.

\section{Network Architecture}
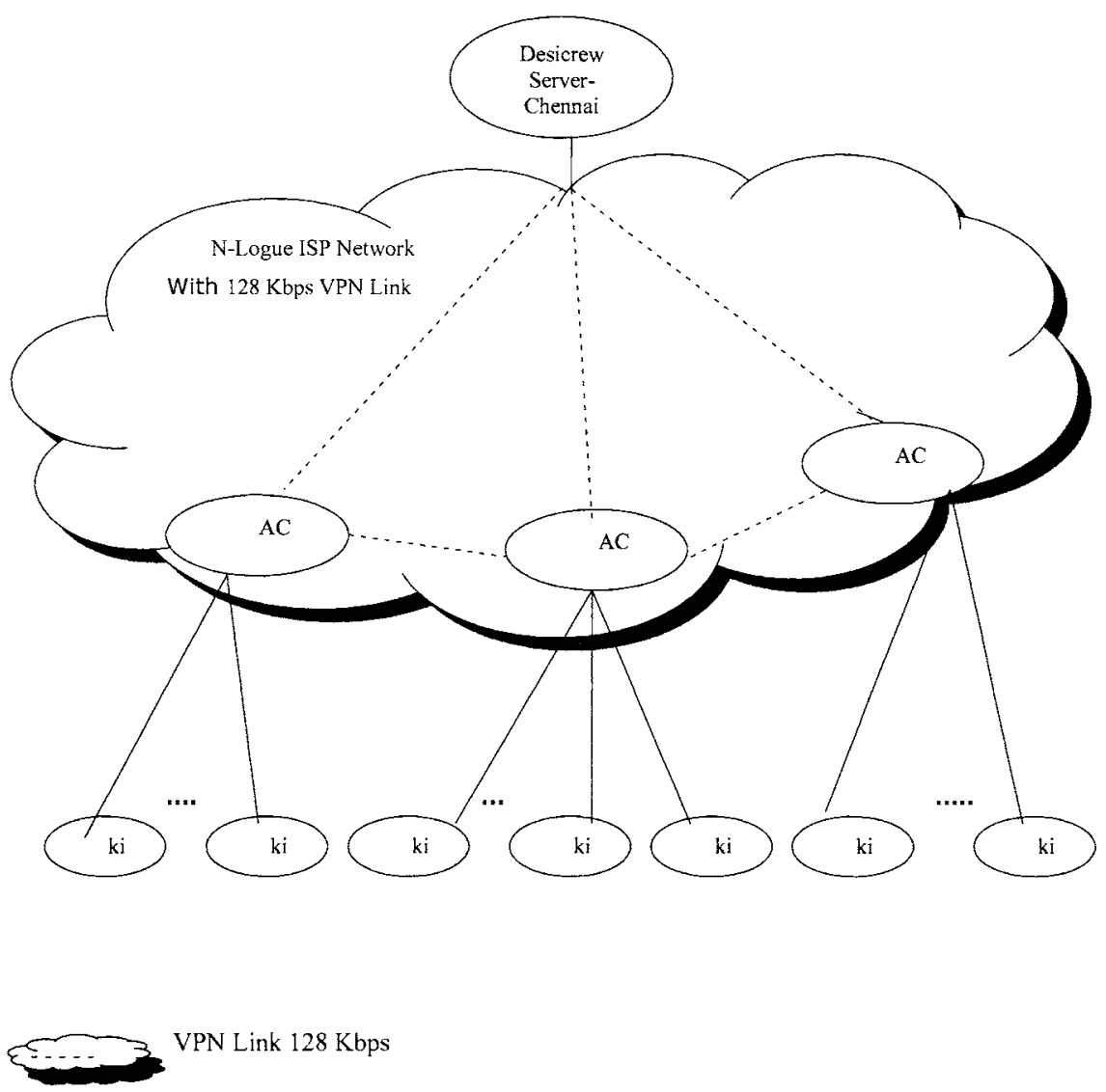

LAN 35-70 Kbps

AC Access Centre

Fig 1: Internet Connectivity used by DesiCrew for Rural BPO 
DesiCrew works closely with a partner, N-Logue communications [6], a rural ISP to reach the connected population in rural areas. N-Logue provides the internet connectivity for the rural BPO centres through the Access Centres located at the district level. Kiosks are internet centres at villages. The Access Centres are connected across in a VPN link provided by the N-Logue. The kiosks at each village are connected to their respective Access Centres through a LAN of 35-70 Kbps bandwidth. DesiCrew uses this infrastructure.

\section{$4 \quad$ Need for Pegasus}

DesiCrew Solutions have taken up the challenge of distributing work from the city to the rural areas where the available skills are seldom utilised. For DesiCrew to do such work requires appropriate processes to distribute work, manage and monitor work, make quality checks, collect work and redistribute to clients.

Given the scenario of a distributed workforce, DesiCrew requires a web-based application to do all of the above. One such application built is Pegasus, a web-based application that supports remote distribution and supervision of work.

Pegasus is an online data entry software where the image of the to-be digitized data appear on the upper half of the browser and the lower half has the form for data entry. The entered data are stored on a central server from where the data is consolidated and sent to the client. The data can also be used for various supervisory reports.

As seen in the image, Pegasus consists of a split screen interface with a digital replica of the image to be entered. The operator is required to look at the image above and enter the data in the HTML replica below. The images and the data entered are stored on a central server for the purpose of security. 


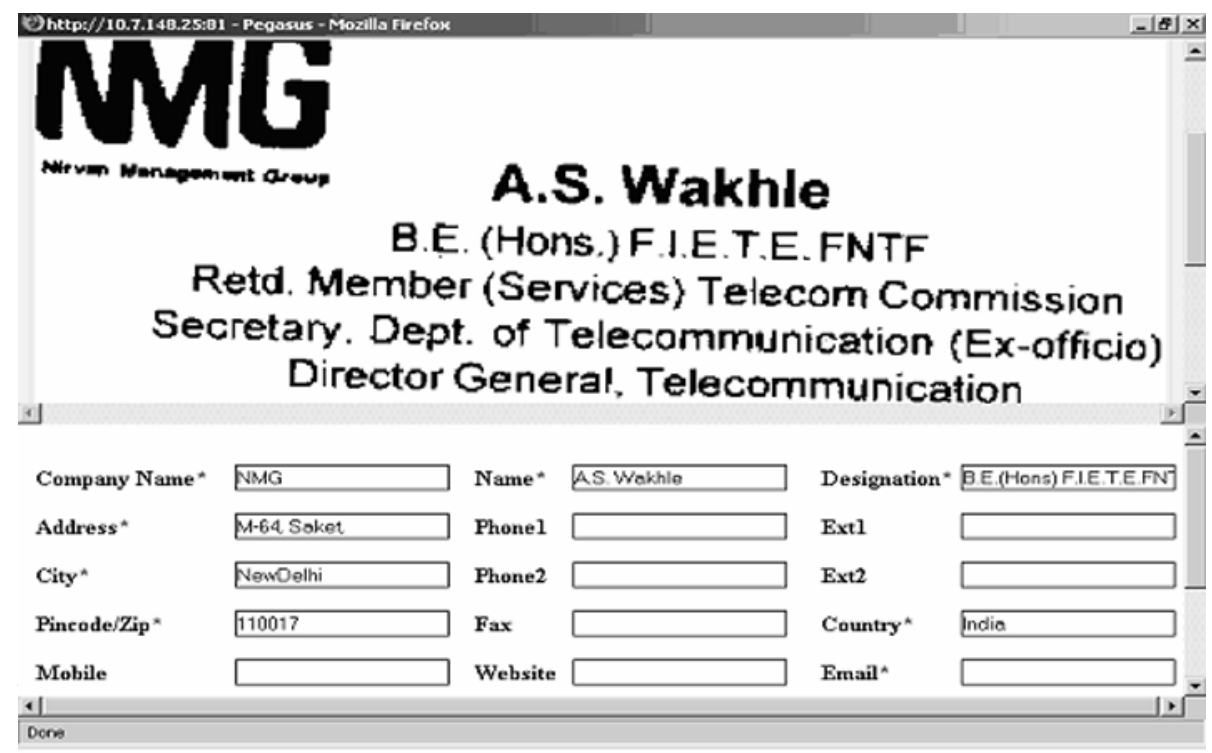

1. Productivity:

(i) Tracks number of forms entered

(ii) No of errors

(iii) Time per form

2. Validation: Field validations such as date, email, phone number, etc.

3. Report Generation

Table 1. Users of the Application, their roles and responsiblities

\begin{tabular}{llll}
\hline S.No. & User & Rights & Roles \& Responsibilities \\
\hline I & First Entry Operator & \multicolumn{1}{c}{ Enter data, View } & Enters Data \\
& & Performance Reports & \\
2 & Second Entry & Enter data, View & Enters Data \\
& Operator & Performance Reports & \\
3 & Coordinator & View mismatches between & Verifies mismatches, Corrects \\
& & First and Second Entry & Data, Specifies error comments \\
& & Data, View His own & \\
& & Performance Reports &
\end{tabular}




\begin{tabular}{llll}
\hline S.No. & User & Rights & Roles \& Responsibilities \\
\hline 4 & Admin & Views all Operator and & Adds Activity, Adds operators to \\
& & Activity Reports, & Activity, Manages and \\
& Add/Delete Activity, & supervises the whole process \\
& Add/Delete Operator &
\end{tabular}

\subsection{Process Flow Diagram}

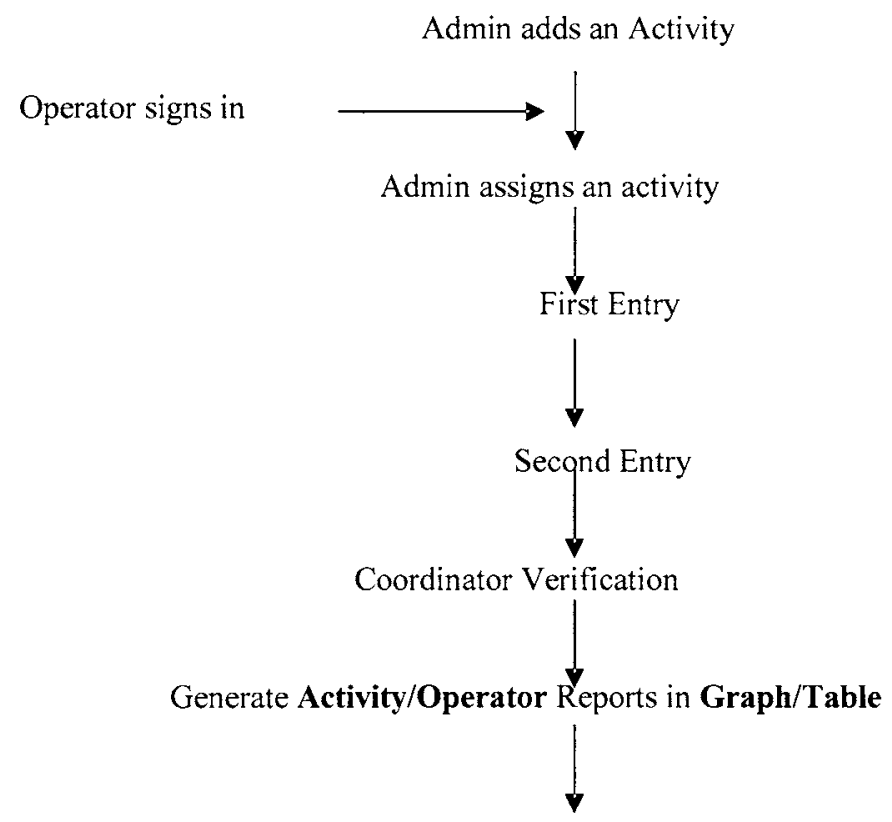

Generate Final Data in Excel sheets

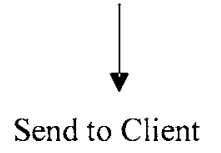

Fig 3: Process Flow of Data Entry Outsourcing

Activity: The work related to each client is defined as an activity. Adding an activity to Pegasus involves specifying duration of the activity, number of operators required,number of forms to be filled, tariff to be followed for payments, uploading of images, etc. Pegasus supports two types of Activities-Regular and Training.

Administrator adds an activity to the Pegasus system. Whenever a new operator signs up, the administrator assigns him to an activity after which the operator is allowed to do dataentry. First, Second and Coordinator entries are done in sequence by the three types of operators. The Final data is now available in the system for generation of reports and the consolidated data is sent to the client. 

Giri

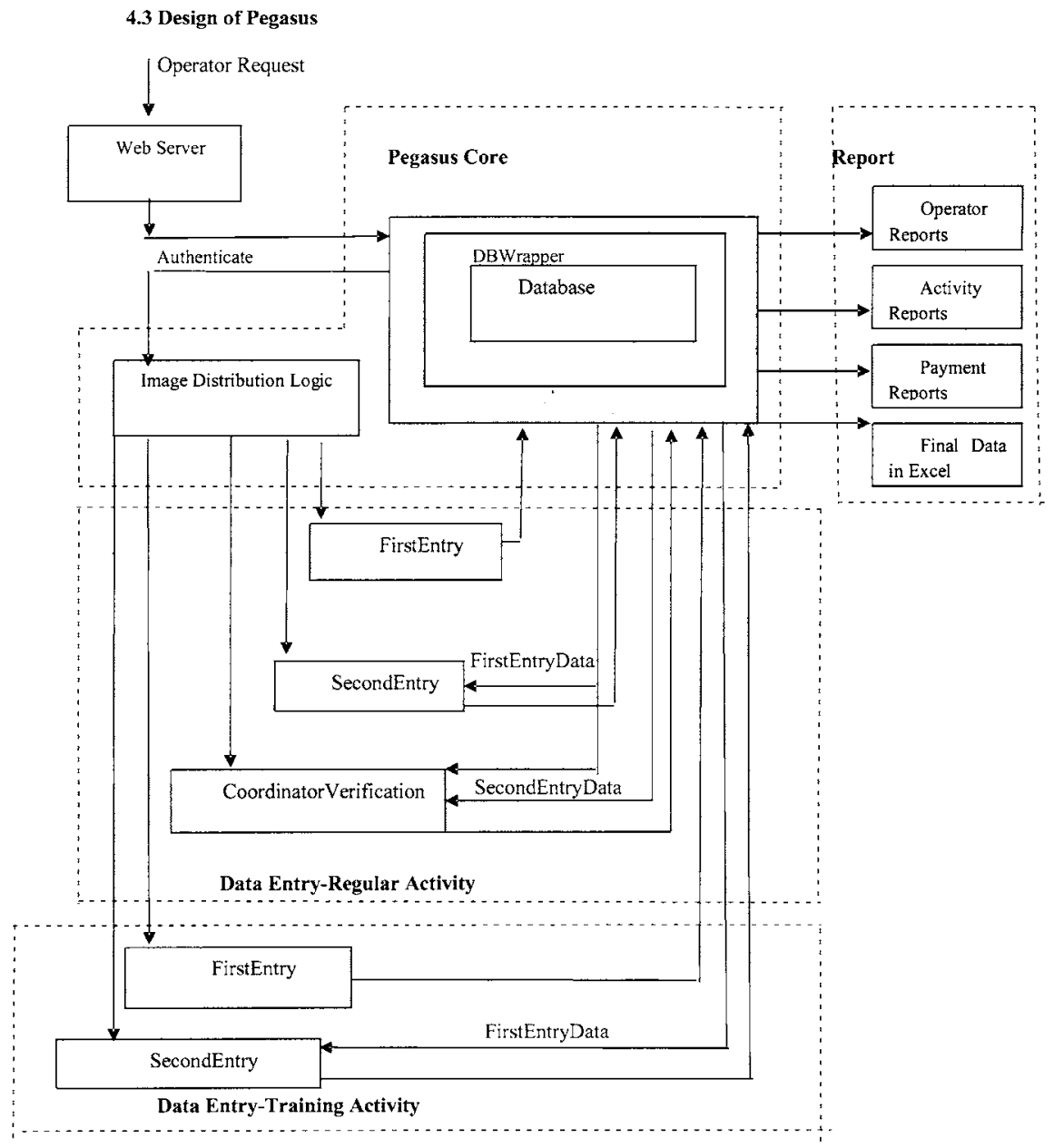

Fig 4: Block Diagram of Pegasus 
Pegasus has a light-weight web services design [7] using fully open-source software. The core is written in PHP and runs on Linux with data stored in Postgresql RDBMS. The user interface runs in a browser on the kiosk PC. It uses AJAX technology [8] for fast response over slow rúral connections.

\subsection{Pegasus Core}

The Pegasus Core comprises of the database which is accessed and manipulated by the APIs through the DBWrapper classes. The Pegasus Core logic handles any user requests for authentication. It also handles expiry of sessions in case of idle time.

\section{ImageDistributionLogic}

Based on the type of operator and the status of an image the Image Distribution Logic fetches the same in the browser. Once the image is accessed it's status is changed such that the same image does not reappear. The Image distribution logic determines which type of operator gets what images based on the image status. An image which has been idle on the browser without being entered is checked and reverted to it's original status.

Download of images is optimized for low bandwidth situations such as $35 \mathrm{kbps}$ by AJAX. The image is also provided with zooming option for clarity on browser.

An additional training module is incorporated in Pegasus wherein the same set of images are distributed across to all authenticated trainees.

The Image distribution logic works in such a way that the same image never appears for another user for a regular activity whereas for a training activity the same set of images appear for all users.

\section{Image Downloading}

The image size is reduced to about $20-40 \mathrm{~KB}$ /page. Over the $35 \mathrm{Kbps}$ link to the kiosk the download time is about 20 seconds. Note that during this time the operator is idle. If the operator takes $30 \mathrm{sec} /$ page to enter the data, she is idle for about $40 \%$ of the time. This affects productivity. We are exploring techniques to reduce this idle time by overlapping the fetching of the next image with the dataentry of one image. Another alternative is is distribution of images on $\mathrm{CD}$. This would require special precaution to preserve the security of data.

\subsection{Data Entry}

Pegasus supports triple data Entry for greater accuracy and reliability of data. The process of Data Entry requires a person to view the image on the browser and type the data in the form provided below. DataEntry Component allows DataEntry in three steps by three different types of operators-FirstEntry, SecondEntry and CoordinatorEntry. This has the 

Giri

First Entry: An operator in this phase enters the data with reference to the image on the screen. The data is transferred to the database. Additionally the DataEntry component records the number of keystrokes, time and duration of updation.

SecondEntry: A second entry operator is allowed to enter the data in the same way as a first entry operator. Additionally this component retrieves the first entry data for this image for the second entry operator for correction incase of mismatches.

Co ordinator Verification: To add to the accuracy of the entered data, the Data Entry Logic also supports a verification by the co ordinator. He is provided with the data entered by the first and second entry operators for each image which has mismatches. The approval/rejection of the data of first and second entry operators by the coordinator results in computation of error percentage which in turn affects the Payout.

Trainee Entry: The first entry for a training activity is done by the administrator. The trainee does the second entry. Pegasus gives the trainee his error reports based on the mismatches from the administrator's entry.

\subsection{Report Generation}

Graphical and Tabular reports are generated for Activity,Operator and Payments Reports.

Operator Reports: Performance reports of each operator can be generated giving information about the number of forms filled, keystrokes and error percentage.

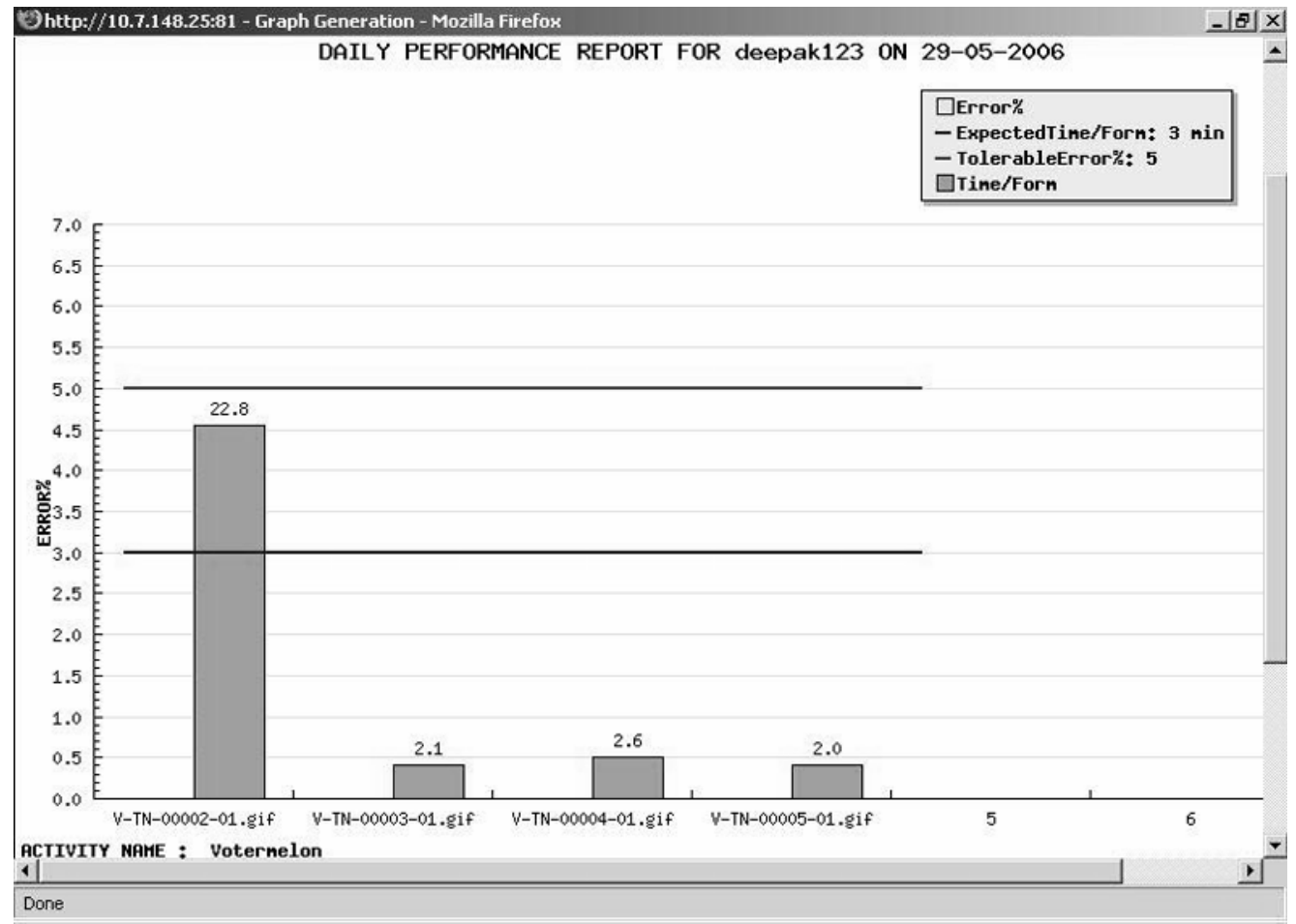

Fig 5: Sample Daily Report of an operator 
Pegasus core does the computation of the error percentage and speed of each operator based on the number of keystrokes.

- Daily Performance Reports

- Weekly Performance Reports

- Overall Performance Reports

- Performance Comparison

Each bar ( See Fig 5) corresponds to each form filled by the operator. For each form filled by the operator the time taken/form and the number of errors are tracked Error \% is calculated and compared with the Tolerable Error\% and time/form with Expected Time/Form.

Activity Reports: Activity Reports (See Fig 6) contain all possible information about the activity such as start and End dates of activity, name of Activity Manager, number of operators working in the activity, status of the activity at any point of time, etc. At every stage the reports provide information about the scheduled activity and the actual status of it.

Payment Reports: Based on the tariff chosen for the activity, number of forms filled by each operator and error percentage payment reports are generated. Payout Module handles two types of payouts-Based on Keystrokes, Based on Forms Filled. It also generates periodic reports about the payments made and to-be made for each operator.

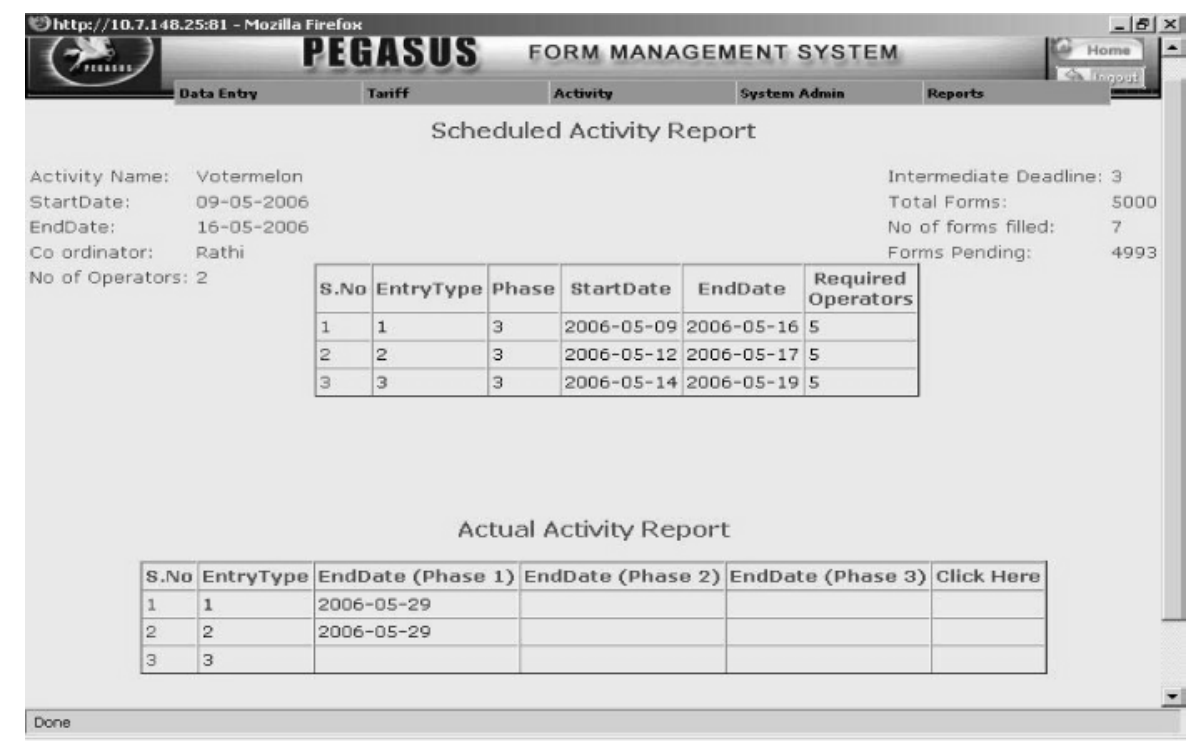

Fig 6: Screenshot of Activity Report

Session Management: Session management is used to keep track of the user logged in to the application. This is heipful to keep track of the operator activity internally

Pegasus is a secure web-based data entry software due to 

Gini

Login: Each user is provided with a login account which helps the administrator to keep track of who accessed the images and when.

VPN: The data transfer is in VPN and cannot be accessed by any intruder/hacker on the Internet.

Restricted Access: The images reside at the server and are not available for downloading /editing those images from any client.

The images are not cached in the browsers and hence a copy of an image at the local machine is not available.

Cut/Copy/Paste/SaveAs options are prevented by disabling the right click on the image.

\section{$5 \quad$ About DesiCrew}

DesiCrew acts as a one point contact for the client and takes responsibility for assuring quality and delivery within the timeline. DesiCrew offers the following services:

Administrative Services: Includes services like digitization and data entry, data collection from secondary sources, data validation

Engineering Services: Covers 2D drafting in AutoCAD and 2D to $3 \mathrm{D}$ modeling in SolidWorks for the manufacturing industry

Content Localization Services: Entails translation of text and voice from English to regional languages (eg Tamil) by schoolteachers and integration with Web and Multimedia mediums

Currently 20 centers with 60 workers are running across Tamil Nadu. Some of these are being set up with the help of Dept of IT and ILFS's CSC Program. DesiCrew is supported and funded by the TeNeT Group at IIT Madras.

\section{The Vision for the Future}

According to Nasscom, the BPO industry in India has experienced a year-on-year growth of 37 per cent in FY 2005-06, and the size of the market is estimated to be $\$ 9-12$ billion as of 2006. It employs around 400,000 people and is expected to face a shortfall of 262,000 professionals by the year 2012 . With some IT training, there is a significant opportunity for the large number of high-school graduates and undergraduate degree-holders in rural areas to fill a part of this gap in the future. The potential of IT-enabled services is much larger than that of manufacturing or other services because it does not involve the physical movement of goods or people.

If the Indian Government's goal of setting up Village Information Centers with connectivity in 100,000 villages is achieved [9], all of these villages would have the potential to act as BPO centers. Consider $10 \%$ of these villages employing 20 people at an average salary of $\$ 65$ per month. This would directly add $\$ 1.56$ billion to the rural economy per annum and create 200,000 jobs. The demand for several allied industries such as hardware servicing and software development would also grow.

The Rural BPO team envisions playing a significant role in this IT revolution, by leveraging the power of computing and connectivity to create wealth in rural areas. 
The team intends to build capacity in villages, create the right systems and networks for remote business operations and ensure the highest level of professionalism and quality standards. The group believes that this is not just a significant business opportunity, but also a means to catapult the pace of rural development in the country under a wholly different paradigm.

\section{References}

1. Thomas Friedman, The World is Flat, Farrar, Straus and Giroux, April 2005.

2. http://www.ocac.in/csc/Default.asp

3. http://tenet.res.in/

4. http://ites.tenet.res.in/

5. http://desicrew.in/

6. http://www.n-logue.com/

7. T.A. Gonsalves, "Web Services For Rural India", CSI Communications, Vol 29, No 9, March 2006, PP 21-23.

8. http://www.modernmethod.com/*sajax, http://en.wikipedia.org/wiki/AJAX

9. Source For VIC - 1,00,000 - Mission 2007

10. Rural BPOs $14 D$, Leading International magazine on ICT for Development, September 2006 issue on ICTs and Small Medium Business 